\title{
Self-Binding Transition in Bose Condensates with Laser-Induced "Gravitation"
}

\author{
S. Giovanazzi, D. O'Dell, G. Kurizki \\ Department of Chemical Physics, The Weizmann Institute of Science, 76100 Rehovot, Israel
}

(November 17, 2018)

In our recent publication (D. O'Dell, et al, Phys. Rev. Lett. 84, $5687(2000)$ ) we proposed a scheme for electromagnetically generating a self-bound Bose-Einstein condensate with $1 / \mathrm{r}$ attractive interactions: the analog of a Bose star. Here we focus upon the conditions neccessary to observe the transition from external trapping to self-binding. This transition becomes manifest in a sharp reduction of the condensate radius and its dependence on the laser intensity rather that the trap potential.

\section{INTRODUCTION}

We have recently proposed [1] a scheme for inducing a $1 / r$ gravitational-like attractive interatomic potential in an atomic Bose-Einstein condensate (BEC) [2] contained in the near-zone volume of intersecting triads of orthogonal laser beams. For sufficiently strong self-"gravitation" the BEC becomes self-bound. In this unique, novel regime the $1 / r$ attraction balances the outward pressure due to the zero point kinetic energy and the short range s-wave scattering. Here we focus upon the transition from external trapping to self-binding. This transition becomes manifest in a sharp reduction of the condensate radius and its dependence on the laser intensity rather than the trap potential. We analyze the conditions for the observability of the self-binding transition: the threshold laser intensity (Sec. III), the bounds on the number of atoms imposed by the near-zone condition (Sec. III), as well as the loss rates (Sec. IV). Sec. V summarizes the findings.

\section{SELF-BINDING THRESHOLD INTENSITY}

\section{A. Threshold condition}

We need to find a situation where the mean-field self"gravitation" energy associated with the near-zone laserinduced attractive $1 / r$ potential can become (at least) comparable with the short-range s-wave scattering energy. To this end, we examine the mean-field solution for a condensate of atoms interacting via Thirunamachandran's isotropic two-atom potential [3, obtained by directional averaging of the laser-induced dipole-dipole potential. This potential has the form

$$
\begin{aligned}
U_{\text {iso }}(\tilde{r})= & -\frac{15 \pi u}{11 \lambda_{\mathrm{L}}}\left(\frac{\sin (4 \pi \tilde{r})}{(2 \pi \tilde{r})^{2}}+2 \frac{\cos (4 \pi \tilde{r})}{(2 \pi \tilde{r})^{3}}-\right. \\
& \left.-5 \frac{\sin (4 \pi \tilde{r})}{(2 \pi \tilde{r})^{4}}-6 \frac{\cos (4 \pi \tilde{r})}{(2 \pi \tilde{r})^{5}}+3 \frac{\sin (4 \pi \tilde{r})}{(2 \pi \tilde{r})^{6}}\right)
\end{aligned}
$$

where $\tilde{r}=r / \lambda_{\mathrm{L}}$, is normalized to the laser wavelength $\lambda_{\mathrm{L}}$, and

$$
u=(11 \pi / 15)\left(I \alpha^{2} / c \epsilon_{0}^{2} \lambda_{\mathrm{L}}^{2}\right)
$$

$I$ being the sum of the intensities of all the lasers, and $\alpha$ the atomic polarizability. The potential begins to oscillate (i.e. becomes alternatingly repulsive and attractive) at distances beyond $\sim 0.36 \lambda_{\mathrm{L}}$. However, this potential can support a self-bound condensate with a larger radius as shown below.

We use the mean-field approximation (MFA), as embodied in the following generalized Gross-Pitaevskii equation [1], to calculate the ground-state order parameter $\Psi(\mathbf{R})$ of a BEC subject to a laser-induced interatomic interaction

$$
\mu \Psi(\mathbf{R})=\left[-\frac{\hbar^{2}}{2 m} \nabla^{2}+V_{\text {ext }}(\mathbf{R})+V_{\mathrm{sc}}(\mathbf{R})\right] \Psi(\mathbf{R})
$$

where $m$ is the atomic mass, $V_{\text {ext }}(R)=m \omega_{0}^{2} R^{2} / 2$ is an isotropic external trap potential (which will be considered negligible - see below), and $V_{\mathrm{sc}}(\mathbf{R})$ is the selfconsistent potential

$$
V_{\mathrm{sc}}(\mathbf{R})=g \rho(\mathbf{R})+\int d^{3} R^{\prime} U_{\mathrm{iso}}\left(\mathbf{R}^{\prime}-\mathbf{R}\right) \rho\left(\mathbf{R}^{\prime}\right)
$$

where $\rho(\mathbf{R})=\Psi^{2}(\mathbf{R})$ is the density and $g=4 \pi a \hbar^{2} / m, a$ being the s-wave scattering length.

In cold dilute atomic BECs with short-range s-wave scattering, the validity of the MFA (i.e. the GrossPitaevskii equation [4]), is well established providing $\rho a^{3} \ll 1$. However, the MFA is also valid for the long range repulsive coulomb potential, $+u / r$, provided many atoms lie within an interaction sphere with a Bohr-type radius, $a_{*}=h^{2} / m u$, so that $\rho a_{*}^{3} \gg 1$ [5]. This condition means that the potential must be weak. Remarkably, self-gravitating BECs simultaneously satisfy both of these MFA validity conditions as can be readily verified using the ensuing expressions.

The condensate radius can be studied using the variational wavefunction $\Psi_{w}(R)=\sqrt{N} \exp \left(-R^{2} / 2 w^{2} \lambda_{\mathrm{L}}^{2}\right) /$ $\left(\pi w^{2} \lambda_{\mathrm{L}}^{2}\right)^{\frac{3}{4}}$, where $w$ is a dimensionless variational parameter giving the width of the condensate. The variational solution in the limit of negligible kinetic energy (ThomasFermi limit) yields a self-bound condensate, i.e. finite $w$ 
(see Fig. 1 and Fig. 2 below), if the laser intensity exceeds the following threshold value (in S.I. units)

$$
I_{0}=\frac{48 \pi}{7} \frac{\hbar^{2} c \epsilon_{0}^{2}}{m \alpha^{2}} a .
$$

Here $I_{0}$ is the total intensity supplied by all the laser beams: for a triad each laser should have $1 / 3$ of the above value and for the 6 triad configuration |1 12 of the lasers should have $1 / 15$, and the remaining 6 should have $1 / 30$, of the above value. The threshold $I_{0}$ signifies the equality of the gravitational-like potential and the s-wave scattering potential.

With an intensity 1.5 times the threshold value (Eq. (5)) (arrow in Fig 2) the expectation value of the rms condensate radius $R_{r m s}=\sqrt{\left\langle R^{2}\right\rangle}$ is a fraction of the laser wavelength $\lambda_{\mathrm{L}}\left(R_{r m s} \approx 0.43 \times \lambda_{\mathrm{L}}\right)$. The condensate is less and less confined as one approaches the threshold (5) - see Fig. 2, from above. Increasing the intensity $I$ reduces the condensate radius which becomes, in the asymptotic limit, proportional to $1 / \sqrt{I}$. Thus the dependence $R_{r m s} \sim\left(I_{0} / I\right)^{1 / 2} \lambda_{\mathrm{L}}$ is a distinct experimental signature of self-binding.

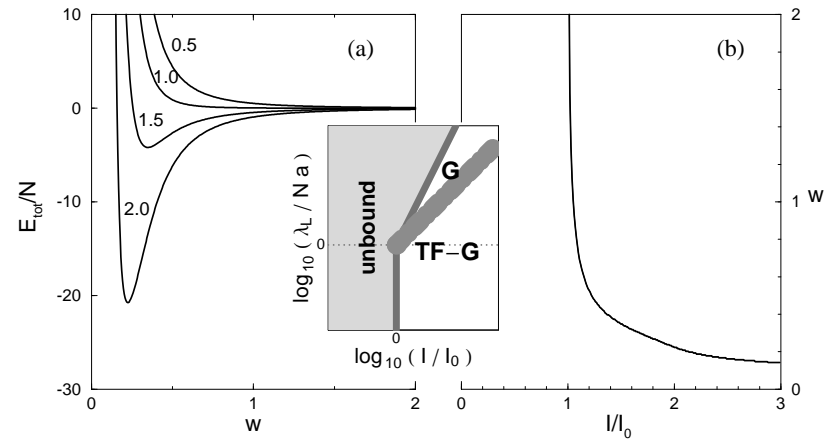

FIG. 1. (a) Variational mean field energies per particles in the case of negligible kinetic energy (TF-G regime) and $\lambda_{\mathrm{L}} / N a \ll 1$ plotted versus the trial size $w$ for different values of $I / I_{0}$. (b) Equilibrium value of $w$ versus $I / I_{0}$ in the limit of negligible kinetic energy (Thomas-Fermi limit). Only for $I / I_{0}>1$ are self-bound variational solutions (having minimum at finite $w$ ) observed. Inset - Schematic phase portrait of the transition from unbound to self-bound regime for negligible external trapping is plotted versus $\log _{10}\left(\lambda_{\mathrm{L}} / N a\right)$ and $\log _{10}\left(I / I_{0}\right)$.

At the threshold intensity an external harmonic trap becomes negligible when $\rho l_{0} \lambda_{\mathrm{L}} a \gg 1$, where $l_{0}^{2}=\hbar / m \omega_{0}$ and $\rho$ is the density. As the laser intensity is increased beyond this value the trap becomes increasingly "irrelevant" - it is not necessary to turn it off to access the TF-G regime, where $r^{-1}$ and s-wave scattering dominate.

The threshold $I_{0}(5)$ is evaluated neglecting the kinetic energy. The role of kinetic energy can be discussed in terms of $\lambda_{\mathrm{L}} / \mathrm{Na}$ (approximately the ratio between the kinetic energy $N \hbar^{2} / m \lambda_{\mathrm{L}}^{2}$ and the scattering energy
$N^{2} \hbar^{2} a / m \lambda_{\mathrm{L}}^{3}$, as shown schematically in the phase portrait in Fig. 1 (drawn for negligible external trapping) which can modify the threshold for self-binding. The G regime, representing the purely "gravitational" counterpart of the TF-G regime, where only "self-gravitation" and kinetic energy play a role [1] (as in a Bose star [6]) is accessed when

$$
\frac{\lambda_{\mathrm{L}}}{N a} \lesssim \frac{I}{I_{0}} \lesssim\left(\frac{\lambda_{\mathrm{L}}}{N a}\right)^{2}
$$

that implies $1 \lesssim \lambda_{\mathrm{L}} / N a$.

At this point the variety of choices can be mainly divided into two categories: i) to work with long laser wavelengths in order to contain many atoms within the near zone, at the price of very high threshold power; ii) use laser wavelengths moderately detuned from an atomic resonance, so as to benefit from the increased polarizability, at the price of considerably fewer self-bound atoms.

\section{B. Long-wavelength (static polarizability) threshold}

The threshold intensity (Eq. (5)) is independent of the laser wavelength $\lambda_{\mathrm{L}}$, as long as the dynamic polarizability $\alpha(q)$ is too. The $I_{0}$ threshold takes the following zerofrequency (static) values: $I_{0}=5.65 \times 10^{9} \mathrm{Watts} / \mathrm{cm}^{2}$ for sodium, $I_{0}=8.19 \times 10^{8} \mathrm{Watts} / \mathrm{cm}^{2}$ for rubidium. It is sufficient to use $20 \mathrm{~W} \times 3$ beams of $\mathrm{Nd}$ :Yag lasers focused down to $10 \mu \mathrm{m}$ for rubidium to exceed the threshold. By contrast, we require multi $\mathrm{kW} \mathrm{CO}_{2}$ lasers focussed down to $100 \mu \mathrm{m}$ for the same purpose. A laser beam with a gaussian profile focussed to $10 \lambda_{\mathrm{L}}$ would exert a large inward radial dipole force on each atom, so non-gaussian optics giving a very flat intensity profile [8] over the condensate region may be required in the long-wavelength (static) case. There remains the problem of random noise in the intensity profile, but fortunately this can only exist on scales larger than the wavelength and so may be overcome.

An additional option is to reduce the scattering length $a$ (to which the threshold intensity (5) is proportional). This is possible in the vicinity of (but somewhat off) a Feshbach resonance, as demonstrated experimentally [7]: reduction of $a$, and correspondly $I_{0}$, by one to two orders of magnitude would eliminate the need for non-gaussian optics in the static polarizability case.

\section{Moderate-detuning threshold}

Using a moderate detuning from an atomic resonance one can increase the polarizability by many orders of magnitude compared to its zero frequency value.

In a recent experiment on superradiance [9] the laser was red detuned by $1.7 \mathrm{GHz}$ from the $3 \mathrm{~S}_{1 / 2}, \mathrm{~F}=1 \rightarrow$ $3 \mathrm{P}_{3 / 2}, \mathrm{~F}=0,1,2$, transition of sodium. With this detuning, the polarizability in cgs units is $\alpha=3.534 \times$ 
$10^{-18} \mathrm{~cm}^{3}$, which is $\approx 1.5 \times 10^{5}$ times the static value of the polarizability. The threshold intensity (5) is then reduced by a factor $\approx 2.3 \times 10^{10}$ compared to the static polarizability case, becoming $I_{0} \approx 262 \mathrm{~mW} / \mathrm{cm}^{2}$ for sodium, which is close to the values used in Ref. [9].

With this value of threshold intensity the gradient forces can be negligible if the focal spots of the lasers are much wider than $\lambda_{\mathrm{L}}$.

\section{Moderate-detuning saturation and repulsion}

The potential (11) is the result of a 4 th order, twoatom, QED process [3], valid when the laser is far detuned from any atomic transitions. This means that the initial absorption of a laser photon and the subsequent intermediate steps are virtual processes (which are most significant in the near-zone), followed by photon emission back into the original laser mode. A different process can take place when the laser is on-resonance. Genuine absorption of a laser photon by a single atom (measured by the saturation), followed by spontaneous emission of this real photon is a process that radiates energy. If another atom absorbs this radiation then in the far-zone it feels a repulsive Coulomb-like force $F_{\text {repuls }}=K / r^{2}$ [11, which has been recently measured in rubidium molasses 12. For moderate detuning, can this force counteract our attractive gravitation-like force $F_{\text {grav }}=-u / r^{2}$ ?

For detuning $\delta$ much larger than both the Rabi frequency $\Omega$ and the linewhidth $\gamma$ of the resonance, the saturation parameter $s=I d^{2} /\left(\epsilon_{0} c \hbar^{2} \delta^{2}\right)$ [10], where $d$ is the dipole matrix element, becomes independent of the detuning when calculated at the threshold intensity (5)

$$
s\left(I=I_{0}\right)=\frac{48 \pi}{7} \frac{a \epsilon_{0} \hbar^{2}}{m d^{2}} .
$$

It is then found that 12 2 $K \approx \sigma_{0}^{2} I_{s} \Omega^{4} /\left(16 c \delta^{2}\right)$, where $\sigma_{0}$ is the resonant absorption cross section and $I_{s}$ is the corresponding saturation intensity. On comparing this expression with $u$ (Eq. (2)), we find that, in terms of the saturation parameter $s$,

$$
K \approx s u
$$

For the sodium transition and $1.7 \mathrm{GHz}$ detuning referred above, Eq. (7) yields very small value $s \approx 0.0003$. This implies that under the moderate-detuning conditions discussed above, the repulsive force has a negligible effect on self-binding.

\section{NUMBER OF SELF-BOUND ATOMS}

A key experimental restriction on self-binding is that the atoms should be in the near-zone to feel the $1 / r$ potential: a condensate smaller than the laser wavelength limits the number of atoms involved. Let us assume we have the maximum density of some $10^{15}$ atoms $/ \mathrm{cm}^{3}$. Using the gaussian wavefunction one can have of the order of $10^{6}$ or $10^{3}$ atoms in the condensate irradiated by a $\mathrm{CO}_{2}$ laser or Nd:Yag laser, respectively (see Fig. 2).

The price of moderately detuned wavelengths $(\approx$ $.589 \mu \mathrm{m}$ for sodium) is the small number of atoms involved. With an intensity $I \approx 1.5 I_{0}$ the atom cloud contains $\approx 40$ atoms as the peak density ranges from $10^{15}$ to $10^{16}$ atoms $/ \mathrm{cm}^{3}$. Although this number is small, it is sufficient to demonstrate the self-binding effect.

For given values of $I, \alpha, a$, and $m$, we are either in the $\mathrm{G}$ regime or the TF-G regime, depending on whether the number of atoms $N$ is smaller or larger than the number

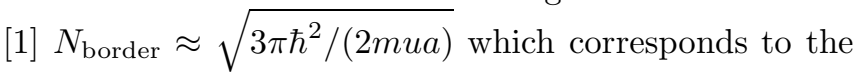
line separating the two regions in the inset of Fig. 1.

It so happens that 40 , the lower estimate of the number of self-bound sodium atoms obtainable in the moderatedetuning regime, is very close to $N_{\text {border }}$. This is an interesting region, because both the kinetic energy and the s-wave scattering are significant and together with the $r^{-1}$ attraction determine the condensate properties.

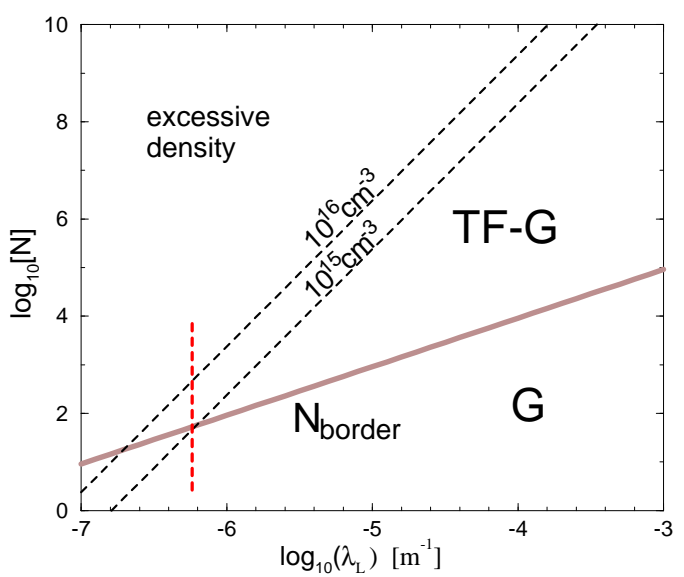

FIG. 2. Range of numbers $N$ of Na condensate atoms as a function of $\lambda_{\mathrm{L}}$ that are compatible with a TF-G or G solution. The density is $10^{15}-10^{16}$ atoms $/ \mathrm{cm}^{3}$ and the intensity is 1.5 times the threshold intensity (5). The region above $10^{16} \mathrm{~cm}^{-3}$ corresponds to excessive density. The vertical long-dashed line corresponds to the moderate-detuning choice discussed for Na.

\section{LOSS RATES}

\section{A. Spontaneous Rayleigh losses}

The single-atom Rayleigh scattering rate $\Gamma_{\text {ray }}$ leads to depletion of the condensate. The probability amplitude for inelastic scattering from the ground state $|0\rangle$ of the near-zone condensate to any excited state $|n\rangle$ due to an external field with wavevector $\mathbf{q}$ is proportional to 
$\sqrt{N} \sum_{n \neq 0}\langle n|(\mathbf{q} \cdot \mathbf{r})| 0\rangle$. Hence, for sample sizes less than a wavelength we expect the spontaneous Rayleigh scattering rate to be reduced by a factor at least as small as $\left(q R_{r m s}\right)^{2}$, analogously to the Lamb-Dicke effect [13]. The lifetime of the condensate, when determined from spontaneous Rayleigh scattering alone, is estimated to be

$$
\tau_{\text {ray }} \geq\left(\Gamma_{\text {ray }}\left(q R_{r m s}\right)^{2}\right)^{-1}
$$

Since $\Gamma_{\text {ray }}=I q^{3} \alpha^{2} /\left(3 h \epsilon_{0}^{2} c\right)$ [3], it can be expressed in terms of the electromagnetically induced energy $U(r)=$ $-u / r$ of a single pair of atoms separated by a distance equal to the wavelength

$$
\Gamma_{\text {ray }}=\left(\frac{20 \pi}{11}\right) \frac{u}{\hbar \lambda_{\mathrm{L}}}
$$

where $u$ is defined in Eq. (2). Using this relation, we can compare the upper bound on the condensate lifetime set by Rayleigh scattering with the time scale of the dynamics, the requirement being that the system exists long enough to equilibriate. In the TF-G and G (self-bound) regions a characteristic time scale for the dynamics is provided by the following "plasma" frequency

$$
\omega_{\mathrm{p}}^{2}=\frac{4 \pi u \rho_{\mathrm{peak}}}{m}
$$

where $\rho_{\text {peak }}$ is the peak density. We can express $\omega_{\mathrm{p}}$ in terms of the recoil energy $E_{\mathrm{R}}=\hbar^{2} q^{2} / 2 m$ ( $q$ being the mean laser wavelength) and the Rayleigh scattering rate $\Gamma_{\text {ray }}$ using Eq. (10)

$$
\omega_{\mathrm{p}} \approx 0.25 \frac{\hbar \Gamma_{\text {ray }}^{2}}{E_{\mathrm{R}}} N^{2} f^{-3 / 2}
$$

where the factor

$$
f=\frac{1}{2}+\sqrt{\frac{1}{4}+\frac{N^{2}}{N_{\text {border }}^{2}}}
$$

is asymptotically equal to 1 in the $G$ region and $N / N_{\text {border }}$ in the TF-G region. It follows from (12) that the characteristic oscillation frequency $\omega_{\mathrm{p}}$ can be much bigger than $\Gamma_{\text {ray }}$, by a factor proportional to $N^{2}$ or $N^{1 / 2}$ in the $\mathrm{G}$ or TF-G region, respectively. Thus the lifetime can be considerably longer than the characteristic time scale of the dynamics.

Even for the small number of 40 sodium atoms in the self-bound moderate-detuning regime $\left(I=1.5 \times I_{0}, \delta=\right.$ $1.7 \mathrm{GHz}$ ), for which the recoil energy is $E_{\mathrm{R}} / \hbar=1.57 \times 10^{5}$ $\mathrm{s}^{-1}$ and $\Gamma_{\text {ray }}=1.58 \times 10^{4} \mathrm{~s}^{-1}$, we find $\omega_{\mathrm{p}} \approx 20 \times \Gamma_{\text {ray }}$. This implies that several oscillation periods of the selfbound condensate can occur within the Rayleigh lifetime.

\section{B. Intereference losses}

We revisit the expressions for the loss rate $\Gamma_{\text {interf }}$ due to multi-beam interference as obtained in [1]. We can express $\Gamma_{\text {interf }}$ in terms of the recoil energy $E_{\mathrm{R}}$ and Rayleigh scattering rate $\Gamma_{\text {ray }}$ as in Sec. IVA

$$
\Gamma_{\text {interf }} \approx 0.05\left(\frac{\hbar \Gamma_{\text {ray }} N}{E_{\mathrm{R}}}\right)^{4} \sqrt{\frac{\hbar \Omega}{E_{\mathrm{R}}}} \Gamma_{\text {ray }} f^{-3}
$$

where $\Omega$ is the relative detuning of beams in the triad. In the example given in Sec. IV A above, $\Gamma_{\text {interf }}$ turns out to be few times bigger than $\Gamma_{\text {ray }}$, when $\Omega$ is chosen to be of the order of $\omega_{\mathrm{p}}$.

\section{CONCLUSIONS}

Our main conclusion is that at least the TF-G selfbound region is experimentally accessible, although such an experiment would be challenging. Moderate detuning is preferable to the longer wavelength case due to the huge enhancement in the polarizability, but it allows the self-binding of few (less that 100) atoms. If the scattering length were reduced via a Feshbach resonance then this would further facilitate the self-trapping of many more atoms using near-infrared lasers.

This work has been supported by the German-Israeli Foundation (GIF).

[1] D. O'Dell, S. Giovanazzi, G. Kurizki, and V. M. Akulin, Phys. Rev. Lett. 84, 5687 (2000); see commentary by J. Anglin, Nature 40629 (2000).

[2] M.H. Anderson et al., Science 269, 198 (1995); C.C. Bradley et al., Phys. Rev. Lett. 75, 1687 (1995); K.B. Davis et al., Phys. Rev. Lett., 75, 3969 (1995). For a recent review of the atomic BEC theory see, e.g., F. Dalfovo, S. Giorgini, L.P. Pitaevskii, and S. Stringari, Rev. Mod. Phys. 71, 463 (1999).

[3] T. Thirunamachandran, Mol. Phys. 40, 393 (1980); D.P. Craig and T. Thirunamachandran, Molecular Quantum Electrodynamics (Academic Press, London, 1984), Sec. 7.12.

[4] L.P. Pitaevskii, Sov. Phys. JETP 13, 451 (1961); E.P. Gross, Nuovo Cimento 20, 454 (1961); J. Math. Phys. 4, 195 (1963)

[5] L.L. Foldy, Phys. Rev. 124, 649 (1961); A.S. Alexandrov and W.H. Beere, Phys. Rev. B 51, 5887 (1995).

[6] R. Ruffini and S. Bonazzola, Phys. Rev. 187, 1767 (1969); G. Ingrosso and D. Grasso and R. Ruffini, Astron. Astrophys. 248, 481 (1991); P. Jetzer, Phys. Rep. 220, 163 (1992).

[7] S. Inouye et al., Nature, 392, 151, (1998); J. Stenger et al., Phys. Rev. Lett., 82, 2422, (1999). 
[8] D. Chen, Z. Wang, and J. R. Leger, Opt. Lett. 20, 663, (1995).

[9] S. Inouye et al., Science, 285, 571, (1999).

[10] C. Cohen-Tannoudji, J. Dupont-Roc and G. Grynberg, Atom-Photon Interactions (Wiley-Interscience, New York, 1992).

[11] T. Walker, D. Sesko, and C. Wiemann, Phys. Rev. Lett. 64, 408 (1990).

[12] L. Pruvoust, I. Serre, H. T. Duong, and J. Jortner, Phys. Rev. A 61, 053408 (2000).

[13] R. H. Dicke, Phys. Rev., 89, 472 (1953). 